Sussman, M. (1954). J. gen. Microbiol. 10, 110-120.

\title{
Synergistic and Antagonistic Interactions between Morphogenetically Deficient Variants of the Slime Mould Dictyostelium discoideum
}

\author{
By M. SUSSMAN \\ Northwestern University, Evanston, Illinois, U.S.A.
}

\begin{abstract}
SUMMARY : A group of variant strains of the slime mould, Dictyostelium discoideum, was collected, all of which cannot accomplish the complete morphogenetic cycle; instead some stop at intermediate stages. They have been termed ' morphogenetically deficient'. In many cases a mixed population of two deficient strains can achieve synergistic development even to the production of normal fruiting structures with viable spores. The nature and intensity of the synergistic response is a function of the proportions of the partners in the mixture. No recombinant types have been found among the spores obtained from the mixtures. The comparative patterns of response have been ascertained when the deficient stocks are paired in all possible combinations. Finally, an antagonistic interaction between two deficient strains is described.
\end{abstract}

The slime moulds are exceptional Protista in that they normally undergo a complex sequence of differentiation and morphogenesis leading to the formation of organized multicellular structures. This development becomes apparent after the stationary growth phase is reached and includes the following stages: Aggregation, during which the individual myxamoebae move toward central collecting points by means of chemotaxis and under the influence of 'initiator' cells (Bonner, 1947; Sussman \& Noel, 1952; Sussman, 1952); Pseudoplasmodium formation, wherein the aggregates are transformed into organized, directed bodies of cells which, in some species, migrate over considerable distances (Raper, 1941); Culmination, involving the conversion of the pseudoplasmodia into sessile fruiting structures with morphologically differentiated spore, stem, and basal disk cells (Raper \& Fennell, 1952). Fig. 1 is a schematic illustration of the cycle.

That this system is open to heritable modification has been demonstrated by the collection of a number of stable variant strains which display aberrant development (Sussman \& Sussman, 1953). These strains fall into two phenotypic classes. The first consists of those that follow the normal developmental sequence up to a point after which a deviant pathway is taken, leading to the formation of drastically aberrant fruitings. The second class, termed 'morphogenetically deficient', grows normally but cannot complete the developmental cycle. For example, some stocks cannot begin aggregation (aggregateless), while others (fruitless) can proceed partly or completely through aggregation but stop at intermediate stages before the emergence of the mature fruiting structure.

It has been found that in many cases a mixed population of two of these deficient variants can achieve a level of morphogenesis significantly higher 
than either partner can accomplish alone, even to the formation of mature fruiting structures with viable spores. In other cases one deficient partner can prevent the other from reaching the stage that it normally attains when alone. The present paper deals with these synergistic and antagonistic interactions and attempts to provide answers to the following questions: $(a)$ What types of interaction are observable? $(b)$ Do the intensity and the nature of the inter-

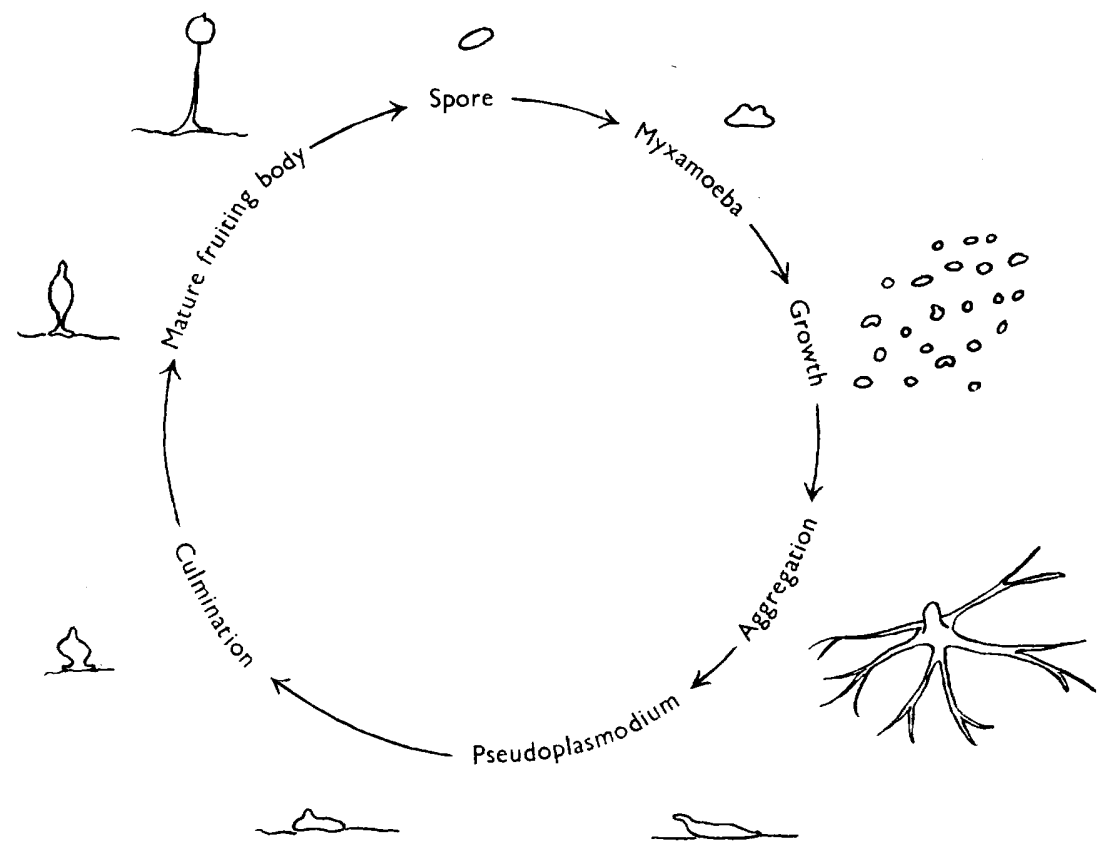

Fig. 1. Schematic diagram of the developmental cycle of Dictyostelium discoideum.

actions depend upon the proportions of the paired cell types? $(c)$ What is the distribution of phenotype among the spores formed by synergistic development? $(d)$ When the deficient stocks are paired in all possible combinations, are distinctive response patterns observable such as might indicate a multiplicity of biochemical alterations leading to the same morphogenetic deficiency?

\section{METHODS}

Organisms. The organism employed in this investigation is representative of Dictyostelium discoideum strain NC-4 and variants obtained therefrom.

Medium. The stocks were carried on glucose peptone agar in association with Aerobacter aerogenes (Sussman, 1951). For mass platings, a few spore masses or a small amount of myxamoebae were spread on a plate with a few drops of bacterial broth culture and incubated at $22^{\circ}$. After 2 days the myxamoebae had grown to the stationary phase, wiping out the bacterial associate, and were ready for experiment. When colonial growth was required, suitably diluted suspensions were dispensed on plates and spread with bacteria. After 3 days plaques of myxamoebae appeared in the area of bacterial growth. 
Assay of developmental ability. The methods used have been described in detail previously (Sussman, 1952). Two-day cultures were harvested and washed by centrifugation and the substantially bacteria-free myxamoebae were suspended in salt solution and counted in a Levy chamber. Suitable dilutions were dispensed in drops on a washed agar distilled water substratum, hereafter designated minimal agar. After the excess fluid had been absorbed by the agar the cells were found to be homogeneously distributed, thus enabling control over the number of cells and the population density. Under these conditions, the viable and total cell counts remain constant, the wild type can aggregate and produce normal fruiting bodies, and the deficient stocks can attain their distinctive levels of development.

\section{RESULTS}

\section{Morphogenetic activity of the deficient strains}

The variants were obtained by ultraviolet treatment in accordance with procedures given previously (Sussman \& Sussman, 1953). In the course of serial passage by clonal platings, some stocks proved to be exceedingly stable while others occasionally threw reverted or mosaic colonies. The extent of development of which these strains were capable were determined in two ways: (1) by examination of the plaques formed on growth medium in the presence of bacteria; (2) by deposition of the washed myxamoebae on minimal agar, after which the plates were incubated and subsequently examined. On these bases the strains fall into four groups. Fig. 2 schematically illustrates the types of development observed on growth medium (column A) and minimal agar (column B). Table 1 contains a listing of the deficient strains according to these groups.

Table 1. Disposition of the morphogenetically deficient strains among the phenotypic groups

$\begin{array}{crcc}\overbrace{\text { I }}^{4} & \text { II } & \text { III } & \text { IV } \\ \overbrace{\text { Agg-53 }}^{\text {Strains }} & & \\ 53-A & \text { Agg-55 } & \text { Fr-4 } & \text { Fr-1 } \\ 57 & 58 & \text { Fr-5 } & \text { Fr-2 } \\ 59 & 200 & \text { Fr-7 } & \text { Fr-3 } \\ 204 & 205 & - & \text { Fr-6 } \\ 206 & 208 & - & - \\ & - & - & -\end{array}$

Group I consists of stocks which are completely aggregateless on growth medium and on minimal agar. Group II includes those strains which produce aggregateless plaques on growth medium but which can aggregate and form normal fruiting bodies on minimal agar. The spores when plated back on growth medium provide clones displaying the original aggregateless phenotype. Evidently, the development is inhibited by something in the complete medium or the associated bacteria. Group III consists of those strains which produce 
fruitless aggregates on minimal agar but which on growth medium form aggregateless plaques. In group IV are the fruitless strains whose morphogenetic activities on growth medium and minimal agar are identical.

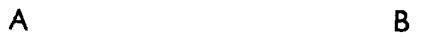

\begin{tabular}{|c|c|c|}
\hline 1 & $\begin{array}{lll}0 & 0 & 0 \\
0 & 0 & 0 \\
0 & 0 & 0 \\
0 & 0 & 0 \\
0 & 0 & 0 \\
00 & 0 & 0 \\
0 & 0 & 0\end{array}$ & $\begin{array}{c}000 \\
00000 \\
00000 \\
00000 \\
000 \\
000\end{array}$ \\
\hline II & $\begin{array}{cccc}0 & 0 & 0 \\
0 & 0 & 0 & 0 \\
0 & 0 & 0 & 0 \\
0 & 0 & 0 & 0 \\
0 & 0 & 0 & 0 \\
0 & 0 & 0 & 0\end{array}$ & $\varphi_{-}^{9}$ \\
\hline III & 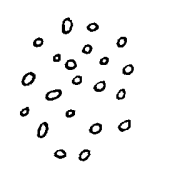 & $\Omega$ \\
\hline \multirow{3}{*}{ IV } & $-\infty-$ & $-\infty \sim$ \\
\hline & $-e^{2} 3-$ & $-e n_{3}$ \\
\hline & $-\Omega$. & $\Omega_{-}$ \\
\hline
\end{tabular}

Fig. 2. Developmental capacities of the four phenotypic groups of deficient variants. Column A refers to the maximum development displayed on growth medium with bacteria; column $\mathrm{B}$ to that displayed by washed cell suspensions on minimal agar.

\section{The morphogenetic activity of mixtures of deficient strains}

The strains were grown and prepared for experiment by procedures described in the Methods section. Appropriate cell mixtures were made and dispensed on minimal agar in replicate $0.01 \mathrm{ml}$. drops. After 24 and $48 \mathrm{hr}$. incubation the plates were examined. As stated previously, many of the combinations achieved significantly greater development than either partner alone. Fig. 3 
illustrates the general types of synergistic development obtained. These are described below:

Normal or thick-stemmed fruits (Fig. 3-A). This development is exemplified by the mixture Fr-2 $\times$ Agg-59. Fr-2, when alone, aggregated to form irregular cell clumps. Agg-59 did not aggregate at all. Together, they produced normal or thick-stemmed fruiting bodies, depending upon the proportions of the cell types. The spores were normal in appearance and were viable.

Bushy fruits (Fig. 3-B). An example of this is the mixture Fr-2 $\times$ Agg-204. Agg-204 (as well as all the others of the 200 series) was obtained, not from the wild type, but from the 'bushy' variant. The latter aggregated normally but the resulting aggregate, which in the wild type was a smooth cone, became heavily papillated. From each papilla emerged a tiny fruiting body with stalk and spore mass, giving the whole the appearance of a bush. When Fr-2 was mixed with Agg-204, the resulting fruiting structure was typically bushy in appearance.

Flat, migrating aggregates (Fig. 3-C). This is exemplified by the mixture Fr-1 $\times$ Agg-53. Fr-1 alone proceeded partly through aggregation, forming a loose flat circular mass of myxamoebae. Agg-53 is a typical aggregateless strain. When Fr-1 was mixed with small numbers of Agg-53 cells, the flat aggregates which were formed migrated at random over the agar surface precisely as do the normal pseudoplasmodia of the wild type. In contrast to the latter, however, the flat migrating aggregate did not produce a fruiting body at the end of its travels.

Partial culminates (Fig. 3-D). This is exemplified by the mixture Fr-1 $\times$ Fr-4. The mixture reached a higher level of development than did either partner alone, but did not proceed as far as the formation of a mature fruiting structure; instead, morphogenetic activity ceased at an intermediate stage.

\section{The dependence of the synergistic response upon the proportions of the partners}

Washed myxamoebae suspensions prepared from the deficient strains were mixed in appropriate dilutions so as to provide a complete range of population ratios. The mixtures were then dispensed on minimal agar in $0.01 \mathrm{ml}$. drops and these were surveyed after 24 and $48 \mathrm{hr}$. incubation. In every case the proportions of the deficient stocks played a vital role in determining the type of interaction and its intensity. Data from two representative combinations are given in Tables 2 and $\mathbf{3}$.

Table 2 shows the results obtained from the mixture, Fr-1 $\times$ Agg-204. When Fr-1 was greatly in excess, no fruiting occurred and one observed only the fruitless aggregates which Fr-1 normally forms when alone. Where the cell numbers were more nearly equal, extensive fruiting occurred. But when the number of Fr-1 cells was quite small, the construction of fruits was only slightly impeded as long as an excess of Agg-204 cells was maintained.

The mixture of Fr-1 $\times$ Agg-53 was capable of two types of synergistic response: $(a)$ the emergence of mature fruiting structures from pseudoplasmodia that do not migrate but were otherwise normal; $(b)$ the formation 
of flat, migrating aggregates that did not fruit. From Table 3 it may be seen that a very high ratio of Fr-1 cells to Agg-53 cells allowed only the appearance
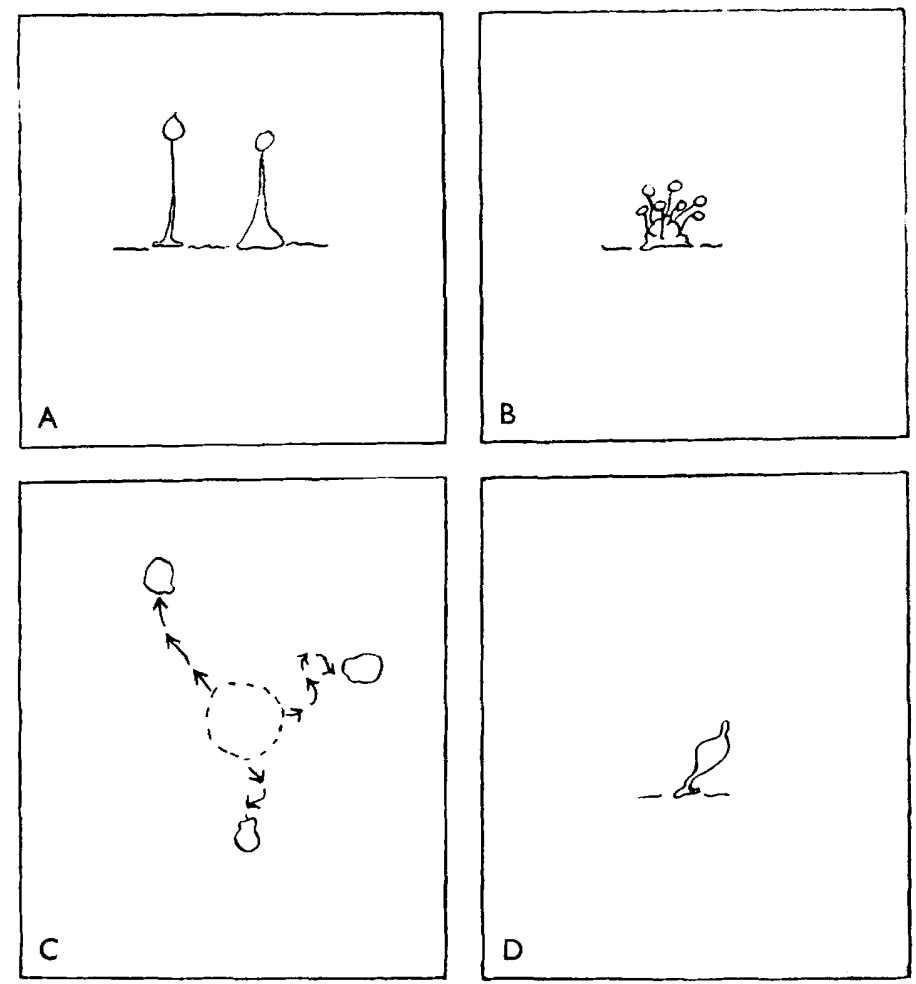

Fig. 3. Schematic illustration of the different types of synergistic development: A-normal and thick-stemmed fruiting bodies; B-bushy fruiting bodies; C--flat migrating aggregates; D-partial culminates.

Table 2. The dependence of the synergism on the proportion of the cell types.

Strain Fr-1 combined with strain Agg-204

Comparative size and number of fruitings scored by number of + signs.

\begin{tabular}{|c|c|c|}
\hline \multicolumn{2}{|c|}{ Strain } & \multirow{3}{*}{$\begin{array}{l}\text { Comparative size and } \\
\text { number of fruitings }\end{array}$} \\
\hline Fr-1 & Agg-204 & \\
\hline \multicolumn{2}{|c|}{ No. cells $\left(\times 10^{-4}\right)$} & \\
\hline 50 & 1 & 0 \\
\hline 50 & 5 & 0 \\
\hline 50 & 13 & $+t+$ \\
\hline 50 & 50 & +++ \\
\hline 13 & 50 & ++++ \\
\hline $\mathbf{5}$ & 50 & +++ \\
\hline 1 & 50 & ++ \\
\hline
\end{tabular}

of the flat migrating aggregates. When that ratio was very low, only mature fruits appeared. Intermediate proportions enabled both types of development to proceed. 
Table 3. The dependence of the synergism on the proportions of the cell types. Strain Fr-1 combined with strain Agg-53

Comparative number of mature fruits and of flat migrating aggregates scored by number of + signs.

\begin{tabular}{|c|c|c|c|}
\hline \multicolumn{2}{|c|}{ Strain } & & \multirow[b]{3}{*}{ Flat migrating } \\
\hline Fr-1 & Agg-53 & & \\
\hline \multicolumn{2}{|c|}{ No. cells $\left(\times 10^{-4}\right)$} & & \\
\hline 100 & 1 & 0 & 0 \\
\hline 100 & 10 & 0 & ++ \\
\hline 100 & 50 & ++ & +++ \\
\hline 100 & 100 & +++ & +++ \\
\hline 50 & 100 & $++t$ & +++ \\
\hline 10 & 100 & +++ & 0 \\
\hline 1 & 100 & ++ & 0 \\
\hline
\end{tabular}

The distribution of phenotype among spores formed during synergistic development

At least two mechanisms might be invoked to explain the synergistic response. One may imagine that the deficient partners exchange metabolic intermediates (syntrophism). The exchange would then enable them to avoid the biochemical blocks that have produced the morphogenetic deficiencies. In this connexion, it may be recalled that Davis (1950) observed a number of such interactions between biochemically deficient strains of Escherichia coli, using growth on minimal medium as the criterion of syntrophism.

A second possibility is that syngamy may be occurring before the onset of aggregation. Morphogenetic sufficiency could then be acquired by the zygotes themselves or by recombinants from subsequent meioses. In an effort to obtain evidence in support of the second possibility, an analysis of the spores from synergistic fruitings was undertaken. The spores were suspended in salt solution, counted in the Levy chamber, and appropriate dilutions were plated with $A$. aerogenes. After 4-5 days of incubation, the resulting clones were surveyed. Total plaque counts revealed a normal percentage of viability among the spores (efficiency of plating $c .75 \%$ ). The differential counts from three representative experiments out of a total of seven are summarized in Table 4.

Table 4. Phenotypic analysis of spores from synergistic fruitings

\begin{tabular}{lrrrrr} 
Combination & \multicolumn{2}{c}{ No. cells $\left(\times 10^{-4}\right)$} & $\begin{array}{c}\text { No. clones } \\
\text { examined }\end{array}$ & $\begin{array}{c}\text { No. aggless } \\
\text { clones }\end{array}$ & $\begin{array}{c}\% \text { aggless } \\
\text { clones }\end{array}$ \\
\cline { 2 - 3 } Fr-1 Agg-204 & 100 & 100 & 778 & 5 & $0 \cdot 6$ \\
& 50 & 50 & 1137 & 11 & $1 \cdot 0$ \\
& 50 & 100 & 126 & 1 & $0 \cdot 8$ \\
Fr-2 Agg-59 & 50 & 50 & 271 & 12 & 4 \\
& 50 & 25 & 203 & 4 & 2 \\
& 50 & 10 & 443 & 0 & 0 \\
Fr-2 Agg-204 & 50 & 100 & 582 & 45 & 8 \\
& 10 & 100 & 86 & 36 & 42 \\
& 5 & 100 & 459 & 308 & 67
\end{tabular}

The plaques proved to be typical in every respect of either of the deficient partners. Not a single wild type or bushy colony or other recombinant type 
was observed. One might argue that the morphogenetically sufficient zygotes or meiotic products do not appear as spores but instead enter the stalk of the fruiting body. R. Sussman (unpublished data), working with the variants under consideration as well as others in a more extensive attempt to demonstrate sexuality in this genus, has found no evidence to support this contention. Thus it must be concluded that in this instance syngamy is either very rare or non-existent.

Table 4 also indicates that the proportions of the two spore types are determined by the particular combination and by the proportions of the myxamoebae originally present. For example, in the combination Fr-1 $\times$ Agg204 , a ratio of 1 of the former to 2 of the latter permitted the appearance of only $0.8 \%$ Agg-204 spores. The same ratio of Fr-2 to Agg-204 allowed 10 times that percentage of Agg-204 spores. Note also that when the ratio of Fr-2 to Agg-204 was decreased from 1:2 to 1:20, the percentage of Agg-204 spores rose from 8 to $67 \%$.

The comparative patterns of synergistic response

Seven fruitless and six aggregateless strains were paired in all possible combinations to determine which pairs reacted synergistically. For each such combination, a sufficient range of population ratios was employed to be certain of the result. Most of the pairs were tested on two independent occasions. Table 5 summarizes the data. In the case of Agg-57, a complete analysis could not be performed because the strain was lost during the course of the experiments. Table 5 reveals that Agg-53, Agg-53 A, and probably Agg-57

Table 5. The comparative response patterns of the deficient strains $x=$ synergistic combinations; $-=$ non-synergistic combinations.

$\begin{array}{lccccccccccccc} & \text { Fr-1 } & \text { Fr-2 } & \text { Fr-3 } & \text { Fr-4 } & \text { Fr-5 } & \text { Fr-6 } & \text { Fr-7 } & 53 & 53-A & 57 & 59 & 204 & 206 \\ \text { Fr-1 } & & - & - & \times & - & \times & \times & \times & \times & \times & \times & \times & - \\ \text { Fr-2 } & - & & - & - & - & - & - & - & - & - & \times & \times & - \\ \text { Fr-3 } & - & - & & - & \times & \times & \times & \times & \times & \times & \times & \times & \times \\ \text { Fr-4 } & \times & - & - & & \times & - & \times & - & - & & - & - & - \\ \text { Fr-5 } & - & - & \times & \times & & - & - & - & - & & - & - & - \\ \text { Fr-6 } & \times & - & \times & - & - & & - & - & - & & - & - & - \\ \text { Fr-7 } & \times & - & \times & \times & - & - & & - & - & & - & - & - \\ \mathbf{5 3} & \times & - & \times & - & - & - & - & & - & - & - & - & - \\ \mathbf{5 3 - A} & \times & - & \times & - & - & - & - & - & & - & - & - & - \\ \mathbf{5 7} & \times & - & \times & & & & & - & - & & - & - & - \\ \mathbf{5 9} & \times & \times & \times & - & - & - & - & - & - & - & & - & - \\ \mathbf{2 0 4} & \times & \times & \times & - & - & - & - & - & - & - & - & & - \\ \mathbf{2 0 6} & - & - & \times & - & - & - & - & - & - & - & - & - & \end{array}$

possess identical patterns of response when paired with the other strains (i.e. all three respond positively with $\mathrm{Fr}-1$ and $\mathrm{Fr}-3$ but no others). The same is true of Agg-59 and Agg-204 (i.e. both respond positively with Fr-1, Fr-2, and Fr-3 but no others). The response of Agg-206 is different from those of the aforementioned groups. Finally, all of the seven fruitless stocks displayed different patterns of response. These results indicate that at least three metabolic alterations can lead to the aggregateless condition while at least seven confer the fruitless character. 
A note of caution is necessary here. The simplest result from an analysis of this kind would be one in which all strains which possessed different deficiencies reacted positively with each other and only those which possessed the same deficiencies should fail to respond in combination. The situation under discussion is complicated by the fact that many strains which display otherwise different response patterns do not react synergistically when combined. Yet it is true that the second criterion is met since no two strains which display otherwise identical patterns respond positively with each other.

The failure to achieve the simplicity inherent in this type of analysis does not invalidate the conclusions reached. One possible explanation is that the test system is insensitive to the point that it does not permit all possible synergistic relations to be effected. Another is that the accumulation of blocked intermediates or their entrance into side reactions creates inhibitory effects which nullify the potential synergism.

A final observation, not immediately interpretable, is the failure of any two aggregateless strains to respond positively when combined.

\section{Attempts to demonstrate exchange of metabolites}

Three kinds of preliminary experiments were performed in an effort to demonstrate exchange; none was successful. The first involved treatment of one deficient stock with a cell-free extract obtained from another deficient strain or the wild type. The second attempted to dissociate the synergistic capacity of either partner from viability by a series of treatments including heating, ultraviolet, and freezing and thawing. In no case could dead cells be shown to react synergistically. The third experimental approach made use of a technique devised by Runyon (1942) who demonstrated the passage of a diffusible chemotactically active substance or substances during aggregation. This worker found that when myxamoebae were allowed to aggregate on agar and underneath a dialysis membrane, other myxamoebae placed on the other side of the membrane become oriented in correspondence with the previously established aggregative centres and streams beneath.

This result was confirmed by the present writer in a similar set of experiments. In addition, it was found that most of the fruitless and at least one of the aggregateless strains so placed above the wild type would so react. But in no case did the development of a deficient stock under these conditions proceed to the formation of fruiting structures. Thus one can be positive that substances were passed from the wild type to the deficient stocks. However, these appear to be substances which normally are exchanged between cells during development and not those which might completely counteract the specific deficiencies.

It is possible that the active intermediates were present in such low concentration and were sufficiently labile that close contact between viable cells was required before the synergistic development could take place. Attempts are under way at present to provide experimental conditions that might conceivably increase the stability. 


\section{An example of antagonistic interactions}

The strain Agg-208 is one that produces aggregateless plaques on growth medium but aggregates and fruits in washed suspension on minimal agar. Agg-206 is a typical aggregateless stock. When the two were mixed on minimal agar, the ability of Agg-208 to aggregate was partly or completely inhibited, depending on the cell proportions. As shown in Table 6, as little as one Agg-206 to ten Agg-208 cells effected complete inhibition. This antagonism is quite specific. No other aggregateless strain tested could inhibit Agg-208 development. Also Agg-206 did not inhibit the development of the wild type, the bushy variant or Agg-58 whose morphogenetic activity is otherwise identical with that of Agg-208. Preliminary experiments with heat killed Agg-206 cells indicated that their viability was not necessary to promote the inhibition.

\section{Table 6. The effect of cell proportions on the antagonism between strain Agg-206 and strain Agg-208}

Extent of aggregation is scored by number of + signs.

\begin{tabular}{|c|c|c|}
\hline \multicolumn{2}{|c|}{ Strain } & \\
\hline Agg-208 & Agg-206 & \\
\hline \multicolumn{3}{|c|}{ No. cells $\left(\times 10^{-4}\right)$} \\
\hline 50 & 50 & 0 \\
\hline 50 & 25 & $\mathbf{0}$ \\
\hline 50 & 5 & 0 \\
\hline 50 & $2 \cdot 5$ & + \\
\hline 50 & 0.5 & $+++t$ \\
\hline 50 & 0 & $+t+t$ \\
\hline 25 & 25 & 0 \\
\hline 25 & 5 & 0 \\
\hline 25 & $2 \cdot \tilde{5}$ & 0 \\
\hline 25 & 0.5 & ++ \\
\hline 25 & 0 & +++ \\
\hline
\end{tabular}

\section{DISCUSSION}

One may envisage at least two opportunities for future investigation that the morphogenetically deficient strains can afford. The first involves an examination of the genetic aspects of the developmental sequence in a manner analogous to that used so successfully with Neurospora crassa. However, to achieve that end a clearly defined syngametic system and a method of segregation analysis must be available, as well as a good deal more detailed biochemical information than is now at hand.

A more immediately encouraging prospect would seem to lie in the direction of determining the identities of the metabolites vital to the morphogenetic cycle and to elucidate the biosynthetic pathways that produce them. It is hoped that further investigation will provide an adequate test system for the demonstration of the active materials and their precursors.

Two possibilities were proposed to explain the synergistic response. One 
involves sexuality and the other the exchange of intermediates in order to avoid the biochemical blocks (syntrophism). A third and less fortunate possibility is that the stocks are deficient because they cannot contribute one or another of the various cell types required for the differentiated assembly of cells. Thus two deficient partners might provide the complete array in concert but not necessarily through exchange of intermediates.

That the developmental sequence does involve passage of at least some substances is clear from the work of Runyon (1942), Bonner (1947), and the results of the membrane experiments reported here. In addition, it has been noted that strains like Fr-1 can aggregate but that none of the myxamoebae therein can be transformed into spores. Yet when mixed with an aggregateless stock, these same cells provide an extremely large proportion of the spore population. It is not readily understandable how this might be contrived except by passage of chemical inductors from the aggregateless cells to the fruitless ones.

This investigation was aided by a grant from the Division of Grants and Fellowships, National Institutes of Health, U.S.A.

\section{REFERENCES}

Bonner, J. T. (1947). Evidence for the formation of cell aggregates by chemotaxis in the development of the slime mold $D$. discoideum. J. exp. Zool. 106, 1.

Davis, B. D. (1950). Studies on nutritionally deficient bacterial mutants isolated with penicillin. Experientia, 6, 41.

RAPER, K. B. (1941). Developmental patterns in simple slime molds. Growth (3rd Symposium), 5, 41.

Raper, K. B. \& Fennell, D. (1952). Stalk formation in Dictyostelium. Bull. Torrey bot. $\mathrm{Cl} .79,25$.

Runyon, E. H. (1942). Aggregation of separate cells of Dictyostelium to form a multicellular body. Collect. Net, 17, 88.

Sussman, M. (1951). The origin of cellular heterogeneity in the slime molds, Dictyosteliaceae. J. exp. Zool. 118, 407.

Sussman, M. \& Noer, E. (1952). Analysis of the aggregation stage in the development of the slime molds Dictyosteliaceae. I. Populational distribution of the capacity to initiate aggregation. Biol. Bull., Woods Hole, 103, 259.

Sussman, M. (1952). Analysis of the aggregation stage in the development of the slime molds Dictyosteliaceae. II. Aggregation by mixtures of the wild type and aggregateless variants. Biol. Bull., Woods Hole, 103, 446.

Sussman, R. R. \& Sussman, M. (1953). Cellular differentiation in Dictyosteliaceae : heritable modifications of the developmental pattern. Ann. N.Y. Acad. Sci. 56, 949 . 